\title{
ANTI-FLEXIBLE BIALGEBRAS
}

\author{
MAFOYA LANDRY DASSOUNDO ${ }^{\star}$, CHENGMING BAI $^{\dagger}$, AND MAHOUTON NORBERT HOUNKONNOU
}

\begin{abstract}
We establish a bialgebra theory for anti-flexible algebras in this paper. We introduce the notion of an anti-flexible bialgebra which is equivalent to a Manin triple of anti-flexible algebras. The study of a special case of anti-flexible bialgebras leads to the introduction of antiflexible Yang-Baxter equation in an anti-flexible algebra which is an analogue of the classical Yang-Baxter equation in a Lie algebra or the associative Yang-Baxter equation in an associative algebra. It is a unexpected consequence that both the anti-flexible Yang-Baxter equation and the associative Yang-Baxter equation have the same form. A skew-symmetric solution of anti-flexible Yang-Baxter equation gives an anti-flexible bialgebra. Finally the notions of an $\mathcal{O}$-operator of an anti-flexible algebra and a pre-anti-flexible algebra are introduced to construct skew-symmetric solutions of anti-flexible Yang-Baxter equation.
\end{abstract}

\section{CONTENTS}

1. Introduction

2. Bimodules and matched pairs of anti-flexible algebras

3. Manin triples of anti-flexible algebras and anti-flexible bialgebras

4. A special class of anti-flexible bialgebras

5. $\quad$ O-operators of anti-flexible algebras and pre-anti-flexible algebras

References

\section{INTRODUCTION}

At first, we recall the definition of a flexible algebra.

Definition 1.1. Let $A$ be a vector space over a field $\mathbb{F}$ equipped with a bilinear product $(x, y) \rightarrow$ $x y$. Set the associator as

$$
(x, y, z)=(x y) z-x(y z), \forall x, y, z \in A .
$$

$A$ is called a flexible algebra if the following identity is satisfied

$$
(x, y, x)=0, \text { or equivalently, }(x y) x=x(y x), \forall x, y \in A .
$$

As a natural generalization of associative algebras, flexible algebras were studied widely. For example, using the solvability and reducibility of the radicals of their underlying Lie algebras, finite-dimensional flexible Lie-admissible algebras were characterized in [5]; any simple strictly power-associative algebra of characteristic prime to 6 of degree greater than 2 is a flexible algebra (14]). Note that the "linearization" of the identity (1.2) gives the following equivalent identity by substituting $x+z$ for $x$ in Eq. (1.2):

$$
(x, y, z)+(z, y, x)=0, \forall x, y, z \in A .
$$

2010 Mathematics Subject Classification. 17A20, 17D25, 16D20, 16T10, 16T15, 16T25.

Key words and phrases. anti-flexible algebra, anti-flexible bialgebra, anti-flexible Yang-Baxter equation, $\mathcal{O}-$ operator. 
It is also natural to consider certain generalization of flexible algebras which leads to the introduction of several classes of nonassociative algebras [17]. In particular, the so-called anti-flexible algebras were introduced as follows.

Definition 1.2. Let $A$ be a vector space equipped with a bilinear product $(x, y) \rightarrow x y$. $A$ is called an anti-flexible algebra if the following identity is satisfied

$$
(x, y, z)=(z, y, x), \text { or equivalently, }(x y) z-x(y z)=(z y) x-z(y x), \forall x, y, z \in A \text {. }
$$

Note that the identity (1.4) means that the associator (1.1) is symmetric in $x, z$ and thus an anti-flexible algebra is also called a center-symmetric algebra in [12] (it is also called a $G_{4}$-associative algebra in [11]). The study of anti-flexible algebras is fruitful, too. For example, simplicity and semi-simplicity of anti-flexible algebras were investigated in [18]; the simple, semisimple (totally) anti-flexible algebras over splitting fields of characteristic different to 2 and 3 were studied and classified in [6, 19, 20]; the primitive structures and prime anti-flexible rings were investigated in [7]; furthermore, it were shown that a simple nearly anti-flexible algebra of characteristic prime to 30 satisfying the identity $(x, x, x)=0$ in which its commutator gives non-nilpotent structure possesses a unity element ([8]).

On the other hand, a bialgebra structure on a given algebraic structure is obtained as a coalgebra structure together which gives the same algebraic structure on the dual space with a set of compatibility conditions between the multiplications and comultiplications. One of the most famous examples of bialgebras is the Lie bialgebra ([9]) and more importantly there have been a lot of bialgebra theories for other algebra structures that essentially follow the approach of Lie bialgebras such as antisymmetric infinitesimal bialgebras ([1], 3]), left-symmetric bialgebras ([2]), alternative D-bialgebras ([10]) and Jordan bialgebras ([21]).

In this paper, we give a bialgebra theory for anti-flexible algebras. We still take a similar approach as of the study on Lie bialgebras, that is, the compatibility condition is still decided by an analogue of Manin triple of Lie algebras, which we call a Manin triple of anti-flexible algebras. The notion of anti-flexible bialgebra is thus introduced as an equivalent structure of a Manin triple of anti-flexible algebras, which is interpreted in terms of matched pairs of anti-flexible algebras. Here the dual bimodule of a bimodule of an anti-flexible algebra plays an important role. We would like to point out that both anti-flexible and associative algebras have the same forms of dual bimodules, which is quite different from other generalizations of associative algebras such as left-symmetric algebras ([回]) or other $G$-associative algebras in [11].

Although to our knowledge, a well-constructed cohomology theory for anti-flexible algebras is unknown yet, we still consider a special case of anti-flexible bialgebras following the study of coboundary Lie bialgebras for Lie algebras ([9]) or coboundary antisymmetric infinitesimal bialgebras for associative algebras (河). The study of such a class of anti-flexible bialgebras also leads to the introduction of anti-flexible Yang-Baxter equation in an anti-flexible equation which is an analogue of the classical Yang-Baxter equation in a Lie algebra or the associative YangBaxter equation in an associative algebra. A skew-symmetric solution of anti-flexible Yang-Baxter equation gives an anti-flexible bialgebra.

There is an unexpected consequence that both the anti-flexible Yang-Baxter equation and the associative Yang-Baxter equation have the same form. It is partly due to the fact that both anti-flexible and associative algebras have the same forms of dual bimodules. Therefore some properties of anti-flexible Yang-Baxter equation can be obtained directly from the corresponding ones of associative Yang-Baxter equation. 
In particular, as for the study on the associative Yang-Baxter equation, in order to obtain skew-symmetric solutions of anti-flexible Yang-Baxter equation, we introduce the notions of an $\mathcal{O}$-operator of an anti-flexible algebra which is an analogue of an $\mathcal{O}$-operator of a Lie algebra introduced by Kupershmidt in [13] as a natural generalization of the classical Yang-Baxter equation in a Lie algebra, and a pre-anti-flexible algebra. The former gives a construction of skew-symmetric solutions of anti-flexible Yang-Baxter equation in a semi-direct product anti-flexible algebra, whereas the latter as a generalization of a dendriform algebra ([15]) gives a bimodule of the associated anti-flexible algebra such that the identity is a natural $\mathcal{O}$-operator associated to it. Therefore a construction of skew-symmetric solutions of anti-flexible Yang-Baxter equation and hence antiflexible bialgebras from pre-anti-flexible algebras is given. Note that from the point of view of operads, pre-anti-flexible algebras are the splitting of anti-flexible algebras (四, [16]).

The paper is organized as follows. In Section 2, we study bimodules and matched pairs of anti-flexible algebras. In particular, we give the dual bimodule of a bimodule of an anti-flexible algebra. In Section 3, we give the notion of a Manin triple of anti-flexible algebras and then interpret it in terms of matched pairs of anti-flexible algebras. The notion of an anti-flexible bialgebra is thus introduced as an equivalent structure of a Manin triple of anti-flexible algebras. In Section 4, we consider the special class of anti-flexible bialgebras which lead to the introduction of anti-flexible Yang-Baxter equation. A skew-symmetric solution of anti-flexible Yang-Baxter equation gives such a anti-flexible bialgebra. In Section 5, we introduce the notions of an $\mathcal{O}_{-}$ operator of an anti-flexible algebra and a pre-anti-flexible algebra. The relationships between them and the anti-flexible Yang-Baxter equation are given. In particular, we give constructions of skew-symmetric solutions of anti-flexible Yang-Baxter equation from $\mathcal{O}$-operators of anti-flexible algebras and pre-anti-flexible algebras.

Throughout this paper, all vector spaces are finite-dimensional over a base field $\mathbb{F}$ whose characteristic is not 2, although many results still hold in the infinite dimension.

\section{Bimodules and matched pairs of anti-Flexible algebras}

In this section, we first introduce the notion of a bimodule of an anti-flexible algebra. Then we study the dual bimodule of a bimodule of an anti-flexible algebra. We also give the notion of a matched pair of anti-flexible algebras.

Definition 2.1. Let $(A, \cdot)$ be an anti-flexible algebra and $V$ be a vector space. Let $l, r: A \rightarrow$ $\operatorname{End}(V)$ be two linear maps. If for any $x, y \in A$,

$$
\begin{gathered}
l(x \cdot y)-l(x) l(y)=r(x) r(y)-r(y \cdot x), \\
l(x) r(y)-r(y) l(x)=l(y) r(x)-r(x) l(y),
\end{gathered}
$$

then it is called a bimodule of $(A, \cdot)$, denoted by $(l, r, V)$. Two bimodules $\left(l_{1}, r_{1}, V_{1}\right)$ and $\left(l_{2}, r_{2}, V_{2}\right)$ of an anti-flexible algebra $A$ is called equivalent if there exists a linear isomorphism $\varphi: V_{1} \rightarrow V_{2}$ satisfying

$$
\varphi l_{1}(x)=l_{2}(x) \varphi, \varphi r_{1}(x)=r_{2}(x) \varphi, \quad \forall x \in A .
$$

Remark 2.2. Note that if both sides of Eqs. (2.1) and (2.2) are zero, then they exactly give the definition of a bimodule of an associative algebra.

Let $(A, \cdot)$ be an anti-flexible algebra. For any $x, y \in A$, let $L_{x}$ and $R_{x}$ denote the left and right multiplication operators respectively, that is, $L_{x}(y)=x y$ and $R_{x}(y)=y x$. Let $L, R: A \rightarrow \operatorname{End}(A)$ be two linear maps with $x \rightarrow L_{x}$ and $x \rightarrow R_{x}$ for any $x \in A$ respectively. 
Example 2.3. Let $(A, \cdot)$ be an anti-flexible algebra. Then $(L, R, A)$ is a bimodule of $(A, \cdot)$, which is called the regular bimodule of $(A, \cdot)$.

Proposition 2.4. Let $(A, \cdot)$ be an anti-flexible algebra and $V$ be a vector space. Let $l, r: A \rightarrow$ $\operatorname{End}(V)$ be two linear maps. Then $(l, r, V)$ is a bimodule of $(A, \cdot)$ if and only if the direct sum $A \oplus V$ of vector spaces is turned into an anti-flexible algebra by defining the multiplication in $A \oplus V$ by

$$
(x+u) *(y+v)=x \cdot y+l(x) v+r(y) u, \quad \forall x, y \in A, u, v \in V .
$$

We call it semi-direct product and denote it by $A \ltimes_{l, r} V$ or simply $A \ltimes V$.

Proof. It is straightforward or follows from Theorem 2.9 as a direct consequence.

It is known that an anti-flexible algebra is a Lie-admissible algebra ([11]).

Proposition 2.5. Let $(A, \cdot)$ be an anti-flexible algebra. Define the commutator by

$$
[x, y]=x \cdot y-y \cdot x, \forall x, y \in A .
$$

Then it is a Lie algebra and we denote it by $(\mathfrak{g}(A),[]$,$) or simply \mathfrak{g}(A)$, which is called the associated Lie algebra of $(A, \cdot)$.

Corollary 2.6. Let $(l, r, V)$ be a bimodule of an anti-flexible algebra $(A, \cdot)$. Then $(l-r, V)$ is a representation of the associated Lie algebra $(\mathfrak{g}(A),[]$,$) .$

Proof. For any $x, y \in A$, we have

$$
\begin{aligned}
{[(l-r)(x),(l-r)(y)] } & =[l(x), l(y)]+[r(x), r(y)]-[l(x), r(y)]-[r(x), l(y)] \\
& =[l(x), l(y)]+[r(x), r(y)]=l(x \cdot y-y \cdot x)-r(x \cdot y-y \cdot x) \\
& =(l-r)([x, y]) .
\end{aligned}
$$

Hence $(l-r, V)$ is a representation of $(\mathfrak{g}(A),[]$,$) .$

Let $(A, \cdot)$ be an anti-flexible algebra. Let $V$ be a vector space and $\alpha: A \rightarrow \operatorname{End}(V)$ be a linear map. Define a linear map $\alpha^{*}: A \rightarrow \operatorname{End}\left(V^{*}\right)$ as

$$
\left\langle\alpha^{*}(x) u^{*}, v\right\rangle=\left\langle u^{*}, \alpha(x) v\right\rangle, \quad \forall x \in A, v \in V, u^{*} \in V^{*},
$$

where $\langle$,$\rangle is the usual pairing between V$ and the dual space $V^{*}$.

Proposition 2.7. Let $(l, r, V)$ be a bimodule of an anti-flexible algebra $(A, \cdot)$. Then $\left(r^{*}, l^{*}, V^{*}\right)$ is bimodule of $(A, \cdot)$.

Proof. For all $x, y \in A, u^{*} \in V^{*}, v \in V$, we have

$$
\begin{aligned}
\left\langle\left(r^{*}(x \cdot y)-r^{*}(x) r^{*}(y)\right) u^{*}, v\right\rangle & =\left\langle u^{*},(r(x \cdot y)-r(y) r(x))(v)\right\rangle=\left\langle u^{*},(l(y) l(x)-l(y \cdot x))(v)\right\rangle \\
& =\left\langle\left(l^{*}(x) l^{*}(y)-l^{*}(y \cdot x)\right) u^{*}, v\right\rangle ; \\
\left\langle\left(l^{*}(x) r^{*}(y)-r^{*}(y) l^{*}(x)\right) u^{*}, v\right\rangle & =\left\langle u^{*},(r(y) l(x)-l(x) r(y))(v)\right\rangle=\left\langle u^{*},(r(x) l(y)-l(y) r(x))(v)\right\rangle \\
& =\left\langle\left(l^{*}(y) r^{*}(x)-r^{*}(x) l^{*}(y)\right) u^{*}, v\right\rangle .
\end{aligned}
$$

Hence $\left(r^{*}, l^{*}, V^{*}\right)$ is bimodule of $(A, \cdot)$.

Remark 2.8. Note that for a bimodule $(l, r, V)$ of an associative algebra, $\left(r^{*}, l^{*}, V^{*}\right)$ is also a bimodule. Therefore, for both associative and anti-flexible algebras, the "dual bimodules" in the above sense have the same form. 
Theorem 2.9. (12]) Let $(A, \cdot)$ and $(B, \circ)$ be two anti-flexible algebras. Suppose that there are four linear maps $l_{A}, r_{A}: A \rightarrow \operatorname{End}(B)$ and $l_{B}, r_{B}: B \rightarrow \operatorname{End}(A)$ such that $\left(l_{A}, r_{A}, B\right)$ and $\left(l_{B}, r_{B}, A\right)$ are bimodules of $(A, \cdot)$ and $(B, \circ)$ respectively, obeying the following relations:

$$
\begin{aligned}
l_{B}(a)(x \cdot y)+ & r_{B}(a)(y \cdot x)-r_{B}\left(l_{A}(x) a\right) y-y \cdot\left(r_{B}(a) x\right)-l_{B}\left(r_{A}(x) a\right) y-\left(l_{B}(a) x\right) \cdot y=0, \\
l_{A}(x)(a \circ b)+ & r_{A}(x)(b \circ a)-r_{A}\left(l_{B}(a) x\right) b-b \circ\left(r_{A}(x) a\right)+l_{A}\left(r_{B}(a) x\right) b-\left(l_{A}(x) a\right) \circ b=0, \\
& y \cdot\left(l_{B}(a) x\right)+\left(r_{B}(a) x\right) \cdot y-\left(r_{B}(a) y\right) \cdot x-l_{B}\left(l_{A}(y) a\right) x \\
& +r_{B}\left(r_{A}(x) a\right) y+l_{B}\left(l_{A}(x) a\right) y-x \cdot\left(l_{B}(a) y\right)-r_{B}\left(r_{A}(y) a\right) x=0 \\
& b \circ\left(l_{A}(x) a\right)+\left(r_{A}(x) a\right) \circ b-\left(r_{A}(x) b\right) \circ a-l_{A}\left(l_{B}(b) x\right) a \\
& +r_{A}\left(r_{B}(a) x\right) b+l_{A}\left(l_{B}(a) x\right) b-a \circ\left(l_{A}(x) b\right)-r_{A}\left(r_{B}(b) x\right) a=0
\end{aligned}
$$

for any $x, y \in A, a, b \in B$. Then there is an anti-flexible algebra structure on $A \oplus B$ given by:

$$
(x+a) *(y+b)=\left(x \cdot y+l_{B}(a) y+r_{B}(b) x\right)+\left(a \circ b+l_{A}(x) b+r_{A}(y) a\right), \quad \forall x, y \in A, a, b \in B .
$$

Conversely, every anti-flexible algebra which is a direct sum of the underlying vector spaces of two subalgebras can be obtained from the above way.

Definition 2.10. Let $(A, \cdot)$ and $(B, \circ)$ be two anti-flexible algebras. Suppose that there are four linear maps $l_{A}, r_{A}: A \rightarrow \operatorname{End}(B)$ and $l_{B}, r_{B}: B \rightarrow \operatorname{End}(A)$ such that $\left(l_{A}, r_{A}, B\right)$ and $\left(l_{B}, r_{B}, A\right)$ are bimodules of $(A, \cdot)$ and $(B, \circ)$ and Eqs. 2.7)-2.10) hold. Then we call the six-tuple $\left(A, B, l_{A}, r_{A}, l_{B}, r_{B}\right)$ a matched pair of anti-flexible algebras. We also denote the anti-flexible algebra defined by Eq. (2.11) by $A \bowtie_{l_{B}, r_{B}}^{l_{A}, r_{A}} B$ or simply by $A \bowtie B$.

\section{MANin TRIPLES OF ANTI-FLEXIBLE ALGEBRAS AND ANTI-FLEXIBLE BIALGEBRAS}

In this section, we introduce the notions of a Manin triple of anti-flexible algebras and an anti-flexible bialgebra. The equivalence between them is interpreted in terms of matched pairs of anti-flexible algebras.

Definition 3.1. A bilinear form $\mathfrak{B}$ on an anti-flexible algebra $(A, \cdot)$ is called invariant if

$$
\mathfrak{B}(x \cdot y, z)=\mathfrak{B}(x, y \cdot z), \quad \forall x, y, z \in A .
$$

Proposition 3.2. Let $(A, \cdot)$ be an anti-flexible algebra. If there is a nondegenerate symmetric invariant bilinear form $\mathfrak{B}$ on $A$, then as bimodules of the anti-flexible algebra $(A, \cdot),(L, R, A)$ and $\left(R^{*}, L^{*}, A^{*}\right)$ are equivalent. Conversely, if as bimodules of an anti-flexible algebra $(A, \cdot),(L, R, A)$ and $\left(R^{*}, L^{*}, A^{*}\right)$ are equivalent, then there exists a nondegenerate invariant bilinear form $\mathfrak{B}$ on A.

Proof. Since $\mathfrak{B}$ is nondegenerate, there exists a linear isomorphism $\varphi: A \rightarrow A^{*}$ defined by

$$
\langle\varphi(x), y\rangle=\mathfrak{B}(x, y), \quad \forall x, y \in A .
$$

Hence for any $x, y, z \in A$, we have

$$
\begin{aligned}
\langle\varphi L(x) y, z\rangle & =\mathfrak{B}(x \cdot y, z)=\mathfrak{B}(z, x \cdot y)=\mathfrak{B}(z \cdot x, y)=\langle\varphi(y), z \cdot x\rangle=\left\langle R^{*}(x) \varphi(y), z\right\rangle ; \\
\langle\varphi R(x) y, z\rangle & =\mathfrak{B}(y \cdot x, z)=\mathfrak{B}(y, x \cdot z)=\langle\varphi(y), x \cdot z\rangle=\left\langle L^{*}(x) \varphi(y), z\right\rangle .
\end{aligned}
$$

Hence $(L, R, A)$ and $\left(R^{*}, L^{*}, A^{*}\right)$ are equivalent. Conversely, by a similar way, we can get the conclusion. 
Definition 3.3. A Manin triple of anti-flexible algebras is a triple of anti-flexible algebras $\left(A, A^{+}, A^{-}\right)$together with a nondegenerate symmetric invariant bilinear form $\mathfrak{B}$ on $A$ such that the following conditions are satisfied.

(a) $A^{+}$and $A^{-}$are anti-flexible subalgebras of $A$;

(b) $A=A^{+} \oplus A^{-}$as vector spaces;

(c) $A^{+}$and $A^{-}$are isotropic with respect to $\mathfrak{B}$, that is, $\mathfrak{B}\left(x_{+}, y_{+}\right)=\mathfrak{B}\left(x_{-}, y_{-}\right)=0$ for any $x_{+}, y_{+} \in A^{+}, x_{-}, y_{-} \in A^{-}$.

A isomorphism between two Manin triples $\left(A, A^{+}, A^{-}\right)$and $\left(B, B^{+}, B^{-}\right)$of anti-flexible algebras is an isomorphism $\varphi: A \rightarrow B$ of anti-flexible algebras such that

$$
\varphi\left(A^{+}\right)=B^{+}, \varphi\left(A^{-}\right)=B^{-}, \mathfrak{B}_{A}(x, y)=\mathfrak{B}_{B}(\varphi(x), \varphi(y)), \forall x, y \in A .
$$

Definition 3.4. Let $(A, \cdot)$ be an anti-flexible algebra. Suppose that "o" is an anti-flexible algebra structure on the dual space $A^{*}$ of $A$ and there is an anti-flexible algebra structure on the direct sum $A \oplus A^{*}$ of the underlying vector spaces of $A$ and $A^{*}$ such that $(A, \cdot)$ and $\left(A^{*}, \circ\right)$ are subalgebras and the natural symmetric bilinear form on $A \oplus A^{*}$ given by

$$
\mathfrak{B}_{d}\left(x+a^{*}, y+b^{*}\right):=\left\langle a^{*}, y\right\rangle+\left\langle x, b^{*}\right\rangle, \forall x, y \in A ; a^{*}, b^{*} \in A^{*},
$$

is invariant, then $\left(A \oplus A^{*}, A, A^{*}\right)$ is called a standard Manin triple of anti-flexible algebras associated to $\mathfrak{B}_{d}$.

Obviously, a standard Manin triple of anti-flexible algebras is a Manin triple of anti-flexible algebras. Conversely, we have

Proposition 3.5. Every Manin triple of anti-flexible algebras is isomorphic to a standard one.

Proof. Since in this case $A^{-}$and $\left(A^{+}\right)^{*}$ are identified by the nondegenerate invariant bilinear form, the anti-flexible algebra structure on $A^{-}$is transferred to $\left(A^{+}\right)^{*}$. Hence the anti-flexible algebra structure on $A^{+} \oplus A^{-}$is transferred to $A^{+} \oplus\left(A^{+}\right)^{*}$. Then the conclusion holds.

Proposition 3.6. Let $(A, \cdot)$ be an anti-flexible algebra. Suppose that there is an anti-flexible algebra structure "o" on the dual space $A^{*}$. Then there exists an anti-flexible algebra structure on the vector space $A \oplus A^{*}$ such that $\left(A \oplus A^{*}, A, A^{*}\right)$ is a standard Manin triple of anti-flexible algebras associated to $\mathfrak{B}_{d}$ defined by $E q$. (3.3) if and only if $\left(A, A^{*}, R^{*}, L_{.}^{*}, R_{\circ}^{*}, L_{\circ}^{*}\right)$ is a matched pair of anti-flexible algebras.

Proof. It follows from the same proof of [3, Theorem 2.2.1].

Proposition 3.7. Let $(A, \cdot)$ be an anti-flexible algebra. Suppose that there exists an anti-flexible algebra structure "॰" on the dual space $A^{*}$. Then $\left(A, A^{*}, R_{.}^{*}, L_{.}^{*}, R_{\circ}^{*}, L_{\circ}^{*}\right)$ is a matched pair of anti-flexible algebras if and only if for any $x, y \in A, a \in A^{*}$,

$$
\begin{gathered}
-R_{\circ}^{*}(a)(x \cdot y)-L_{\circ}^{*}(a)(y \cdot x)+L_{\circ}^{*}\left(R_{.}^{*}(x) a\right) y+y \cdot\left(L_{\circ}^{*}(a) x\right)+R_{\circ}^{*}\left(L_{.}^{*}(x) a\right) y+\left(R_{\circ}^{*}(a) x\right) \cdot y=0, \\
y \cdot\left(R_{\circ}^{*}(a) x\right)-x \cdot\left(R_{\circ}^{*}(a) y\right)+\left(L_{\circ}^{*}(a) x\right) \cdot y-\left(L_{\circ}^{*}(a) y\right) \cdot x \\
+L_{\circ}^{*}\left(L_{.}^{*}(x) a\right) y-R_{\circ}^{*}\left(R_{.}^{*}(y) a\right) x+R_{\circ}^{*}\left(R_{.}^{*}(x) a\right) y-L_{\circ}^{*}\left(L_{.}^{*}(y) a\right) x=0 .
\end{gathered}
$$

Proof. Obviously, Eq. (3.4) is exactly Eq. (2.8) and Eq. (3.5) is exactly Eq. (2.10) in the case $l_{A}=R_{*}^{*}, r_{A}=L_{*}^{*}, l_{B}=l_{A^{*}}=R_{\circ}^{*}, r_{B}=r_{A^{*}}=L_{\circ}^{*}$. For any $x, y \in A, a, b \in A^{*}$, we have:

$$
\begin{aligned}
& \left\langle R_{\circ}^{*}(a)(x \cdot y), b\right\rangle=\left\langle x \cdot y, R_{\circ}(a) b\right\rangle=\langle x \cdot y, b \circ a\rangle=\langle L .(x) y, b \circ a\rangle=\left\langle y, L_{.}^{*}(x)(b \circ a)\right\rangle ; \\
& \left\langle L_{\circ}^{*}(a)(y \cdot x), b\right\rangle=\left\langle y \cdot x, L_{\circ}(a) b\right\rangle=\langle y \cdot x, a \circ b\rangle=\langle R .(x) y, a \circ b\rangle=\left\langle y, R_{.}^{*}(x)(a \circ b)\right\rangle ; \\
& \left\langle L_{\circ}^{*}\left(R_{.}^{*}(x) a\right) y, b\right\rangle=\left\langle y, L_{\circ}\left(R_{.}^{*}(x) a\right) b\right\rangle=\left\langle y,\left(R_{.}^{*}(x) a\right) \circ b\right\rangle ;
\end{aligned}
$$




$$
\begin{aligned}
& \left\langle y \cdot\left(L_{\circ}^{*}(a) x\right), b\right\rangle=\left\langle R .\left(L_{\circ}^{*}(a) x\right) y, b\right\rangle=\left\langle y, R_{.}^{*}\left(L_{\circ}^{*}(a) x\right) b\right\rangle ; \\
& \left\langle R_{\circ}^{*}\left(L_{.}^{*}(x) a\right) y, b\right\rangle=\left\langle y, R_{\circ}\left(L_{.}^{*}(x) a\right) b\right\rangle=\left\langle y, b \circ\left(L_{.}^{*}(x) a\right)\right\rangle ; \\
& \left\langle\left(R_{\circ}^{*}(a) x\right) \cdot y, b\right\rangle=\left\langle L .\left(R_{\circ}^{*}(a) x\right) y, b\right\rangle=\left\langle y, L_{.}^{*}\left(R_{\circ}^{*}(a) x\right) b\right\rangle .
\end{aligned}
$$

Then Eq. (2.7) holds if and only if Eq. (2.8) holds. Similarly, Eq. (2.9) holds if and only if Eq. (2.10) holds. Therefore the conclusion holds.

Let $V$ be a vector space. Let $\sigma: V \otimes V \rightarrow V \otimes V$ be the flip defined as

$$
\sigma(x \otimes y)=y \otimes x, \quad \forall x, y \in V .
$$

Theorem 3.8. Let $(A, \cdot)$ be an anti-flexible algebra. Suppose there is an anti-flexible algebra structure "०" on its dual space $A^{*}$ given by a linear map $\Delta^{*}: A^{*} \otimes A^{*} \rightarrow A^{*}$. Then $\left(A, A^{*}, R_{.}^{*}, L_{.}^{*}, R_{\circ}^{*}, L_{\circ}^{*}\right)$ is a matched pair of anti-flexible algebras if and only if $\Delta: A \rightarrow A \otimes A$ satisfies the following two conditions:

$$
\begin{gathered}
\Delta(x \cdot y)+\sigma \Delta(y \cdot x)=(\sigma(\mathrm{id} \otimes L .(y))+R .(y) \otimes \mathrm{id}) \Delta(x)+(\sigma(R .(x) \otimes \mathrm{id})+\mathrm{id} \otimes L .(x)) \Delta(y), \\
(\sigma(\mathrm{id} \otimes R .(y))-\mathrm{id} \otimes R .(y)-\sigma(L .(y) \otimes \mathrm{id})+L .(y) \otimes \mathrm{id}) \Delta(x)= \\
(\sigma(\mathrm{id} \otimes R .(x))-\mathrm{id} \otimes R .(x)-\sigma(L .(x) \otimes \mathrm{id})+L .(x) \otimes \mathrm{id}) \Delta(y),
\end{gathered}
$$

for any $x, y \in A$.

Proof. For any $x, y \in A$ and any $a, b \in A^{*}$, we have

$$
\begin{aligned}
& \langle\Delta(x \cdot y), a \otimes b\rangle=\langle x \cdot y, a \cdot b\rangle,=\left\langle L_{\circ}^{*}(a)(x \cdot y), b\right\rangle, \\
& \langle\sigma \Delta(y \cdot x), a \otimes b\rangle=\langle y \cdot x, b \circ a\rangle=\left\langle R_{\circ}^{*}(a)(y \cdot x), b\right\rangle, \\
& \langle\sigma(\operatorname{id} \otimes L .(y)) \Delta(x), a \otimes b\rangle=\left\langle x, b \circ\left(L^{*}(y) a\right)\right\rangle=\left\langle R_{\circ}^{*}\left(L_{.}^{*}(y) a\right) x, b\right\rangle, \\
& \langle(R .(y) \otimes \operatorname{id}) \Delta(x), a \otimes b\rangle=\left\langle x,\left(R_{.}^{*}(y) a\right) \circ b\right\rangle=\left\langle L_{\circ}^{*}\left(R^{*}(y) a\right) x, b\right\rangle, \\
& \langle\sigma(R .(x) \otimes \operatorname{id}) \Delta(y), a \otimes b\rangle=\left\langle y,\left(R_{*}^{*}(x) b\right) \circ a\right\rangle=\left\langle\left(R_{\circ}^{*}(a) y\right) \cdot x, b\right\rangle, \\
& \langle(\operatorname{id} \otimes L .(x)) \Delta(y), a \otimes b\rangle=\left\langle y, a \circ\left(L_{.}^{*}(x) b\right)\right\rangle=\left\langle x \cdot\left(L_{\circ}^{*}(a) y\right), b\right\rangle .
\end{aligned}
$$

Then Eq. (3.4) is equivalent to Eq. (3.7). Moreover, we have

$$
\begin{aligned}
& \langle\sigma(\mathrm{id} \otimes R .(y)) \Delta(x), a \otimes b\rangle=\left\langle x, b \circ\left(R_{*}^{*}(y) a\right)\right\rangle=\left\langle R_{\circ}^{*}\left(R_{*}^{*}(y) a\right) x, b\right\rangle, \\
& \langle(\mathrm{id} \otimes R .(y)) \Delta(x), a \otimes b\rangle=\left\langle x, a \circ\left(R_{\cdot}^{*}(y) b\right)\right\rangle=\left\langle\left(L_{\circ}^{*}(a) x\right) \cdot y, b\right\rangle, \\
& \langle\sigma(L .(y) \otimes \mathrm{id}) \Delta(x), a \otimes b\rangle=\left\langle x,\left(L_{*}^{*}(y) b\right) \circ a\right\rangle=\left\langle y \cdot\left(R_{\circ}^{*}(a) x\right), b\right\rangle, \\
& \langle(L .(y) \otimes \mathrm{id}) \Delta(x), a \otimes b\rangle=\left\langle x,\left(L_{.}^{*}(y) a\right) \circ b\right\rangle=\left\langle L_{\circ}^{*}\left(L_{.}^{*}(y) a\right) x, b\right\rangle .
\end{aligned}
$$

Then Eq. (3.5) is equivalent to Eq. (3.8). Hence the conclusion holds.

Remark 3.9. From the symmetry of the anti-flexible algebras $(A, \cdot)$ and $\left(A^{*}, \circ\right)$ in the standard Manin triple of anti-flexible algebras associated to $\mathfrak{B}_{d}$, we also can consider a linear map $\gamma$ : $A^{*} \rightarrow A^{*} \otimes A^{*}$ such that $\gamma^{*}: A \otimes A \rightarrow A$ gives the anti-flexible algebra structure "." on $A$. It is straightforward to show that $\Delta$ satisfies Eqs. (3.7) and (3.8) if and only if $\gamma$ satisfies

$$
\begin{gathered}
\gamma(a \circ b)+\sigma \gamma(b \circ a)=\left(\sigma\left(\mathrm{id} \otimes L_{\circ}(b)\right)+R_{\circ}(b) \otimes \mathrm{id}\right) \gamma(a)+\left(\sigma\left(R_{\circ}(a) \otimes \mathrm{id}\right)+\mathrm{id} \otimes L_{\circ}(a)\right) \gamma(b), \\
\left(\sigma\left(\mathrm{id} \otimes R_{\circ}(b)\right)-\mathrm{id} \otimes R_{\circ}(b)-\sigma\left(L_{\circ}(b) \otimes \mathrm{id}\right)+\left(L_{\circ}(b) \otimes \mathrm{id}\right)\right) \gamma(a)= \\
\left(\left(L_{\circ}(a) \otimes \mathrm{id}\right)-\sigma\left(L_{\circ}(a) \otimes \mathrm{id}\right)+\sigma\left(\mathrm{id} \otimes R_{\circ}(a)\right)-\left(\mathrm{id} \otimes R_{\circ}(a)\right)\right) \gamma(b),
\end{gathered}
$$

for any $a, b \in A^{*}$.

Definition 3.10. Let $(A, \cdot)$ be an anti-flexible algebra. An anti-flexible bialgebra structure on $A$ is a linear map $\Delta: A \rightarrow A \otimes A$ such that

(a) $\Delta^{*}: A^{*} \otimes A^{*} \rightarrow A^{*}$ defines an anti-flexible algebra structure on $A^{*}$;

(b) $\Delta$ satisfies Eqs. (3.7) and (3.8). 
We denote it by $(A, \Delta)$ or $\left(A, A^{*}\right)$.

Example 3.11. Let $(A, \Delta)$ be an anti-flexible bialgebra on an anti-flexible algebra $A$. Then $\left(A^{*}, \gamma\right)$ is an anti-flexible bialgebra on the anti-flexible algebra $A^{*}$, where $\gamma$ is given in Remark 3.9.

Combining Proposition 3.6 and Theorem 3.8 together, we have the following conclusion.

Theorem 3.12. Let $(A, \cdot)$ be an anti-flexible algebra. Suppose that there is an anti-flexible algebra structure on its dual space $A^{*}$ denoted "o" which is defined by a linear map $\Delta: A \rightarrow A \otimes A$. Then the following conditions are equivalent.

(a) $\left(A \oplus A^{*}, A, A^{*}\right)$ is a standard Manin triple of anti-flexible algebras associated to $\mathfrak{B}_{d}$ defined by Eq. (3.3).

(b) $\left(A, A^{*}, R_{.}^{*}, L_{.}^{*}, R_{\circ}^{*}, L_{\circ}^{*}\right)$ is a matched pair of anti-flexible algebras.

(c) $(A, \Delta)$ is an anti-flexible bialgebra.

Recall a Lie bialgebra structure on a Lie algebra $\mathfrak{g}$ is a linear map $\delta: \mathfrak{g} \rightarrow \mathfrak{g} \otimes \mathfrak{g}$ such that $\delta^{*}: \mathfrak{g}^{*} \otimes \mathfrak{g}^{*} \rightarrow \mathfrak{g}^{*}$ defines a Lie algebra structure on $\mathfrak{g}^{*}$ and $\delta$ satisfies

$$
\delta[x, y]=(\operatorname{ad}(x) \otimes \mathrm{id}+\mathrm{id} \otimes \operatorname{ad}(x)) \delta(y)-(\operatorname{ad}(y) \otimes \mathrm{id}+\mathrm{id} \otimes \operatorname{ad}(y)) \delta(x), \quad \forall x, y \in \mathfrak{g},
$$

where $\operatorname{ad}(x)(y)=[x, y]$ for any $x, y \in \mathfrak{g}$. We denoted it by $(\mathfrak{g}, \delta)$.

Proposition 3.13. Let $(A, \Delta)$ be an anti-flexible bialgebra. Then $(\mathfrak{g}(A), \delta)$ is a Lie bialgebra, where $\delta=\Delta-\sigma \Delta$.

Proof. It is straightforward.

\section{A special Class of anti-FleXible Bialgebras}

In this section, we consider a special class of anti-flexible bialgebras, that is, the anti-flexible bialgebra $(A, \Delta)$ on an anti-flexible algebra $(A, \cdot)$, with the linear map $\Delta$ defined by

$$
\Delta(x)=(\mathrm{id} \otimes L .(x)) \mathrm{r}+(R .(x) \otimes \mathrm{id}) \sigma \mathrm{r}, \quad \forall x \in A,
$$

where $\mathrm{r} \in A \otimes A$.

Lemma 4.1. Let $(A, \cdot)$ be an anti-flexible algebra and $\mathrm{r} \in A \otimes A$. Let $\Delta: A \rightarrow A \otimes A$ be a linear map defined by Eq. (4.1). Then

$$
\sigma \Delta(x)=(L .(x) \otimes \mathrm{id}) \sigma \mathrm{r}+(\mathrm{id} \otimes R .(x)) \mathrm{r}, \quad \forall x \in A .
$$

Proof. It is straightforward.

Proposition 4.2. Let $(A, \cdot)$ be an anti-flexible algebra and $\mathrm{r} \in A \otimes A$. Let $\Delta: A \rightarrow A \otimes A$ be a linear map defined by Eq. (4.1).

(a) Eq. (3.7) holds if and only if

$$
(L .(x) \otimes R .(y)+R .(x) \otimes L .(y))(\mathrm{r}+\sigma \mathrm{r})=0, \quad \forall x, y \in A .
$$

(b) Eq. (3.8) holds if and only if

$$
(R .(x) \otimes R .(y)-R .(y) \otimes R .(x)+L .(x) \otimes L .(y)-L .(y) \otimes L .(x))(\mathrm{r}+\sigma \mathrm{r})=0, \quad \forall x, y \in A .
$$

Proof. (a) Let $x, y \in A$. By Lemma 4.1, we have

$$
\Delta(x \cdot y)+\sigma \Delta(y \cdot x)=(\operatorname{id} \otimes(L .(x \cdot y)+R .(y \cdot x))) \mathrm{r}+((R .(x \cdot y)+L .(y \cdot x)) \otimes \mathrm{id}) \sigma \mathrm{r} .
$$

By the definition of an anti-flexible algebra, we have

$$
\Delta(x \cdot y)+\sigma \Delta(y \cdot x)=(\mathrm{id} \otimes(L .(x) L .(y)+R .(x) R .(y))) \mathrm{r}+((R .(y) R .(x)+L .(y) L .(x)) \otimes \mathrm{id}) \sigma \mathrm{r} .
$$


Moreover, we have

$$
\begin{aligned}
\sigma(\mathrm{id} \otimes L .(y)) \Delta(x) & =\sigma(\mathrm{id} \otimes L .(y))(\mathrm{id} \otimes L .(x)) \mathrm{r}+\sigma(\mathrm{id} \otimes L .(y))(R .(x) \otimes \mathrm{id}) \sigma \mathrm{r} \\
& =(L .(y) L .(x) \otimes \mathrm{id}) \sigma \mathrm{r}+(L .(y) \otimes \mathrm{id})(\mathrm{id} \otimes R .(x)) \mathrm{r}, \\
(R .(y) \otimes \mathrm{id}) \Delta(x) & =(R .(y) \otimes \mathrm{id})(\mathrm{id} \otimes L .(x)) \mathrm{r}+(R .(y) \otimes \mathrm{id})(R .(x) \otimes \mathrm{id}) \sigma \mathrm{r} \\
& =(R .(y) \otimes \mathrm{id})(\mathrm{id} \otimes L .(x)) \mathrm{r}+(R .(y) R .(x) \otimes \mathrm{id}) \sigma \mathrm{r}, \\
\sigma(R .(x) \otimes \mathrm{id}) \Delta(y) & =\sigma(R .(x) \otimes \mathrm{id})(\mathrm{id} \otimes L .(y)) \mathrm{r}+\sigma(R .(x) \otimes \mathrm{id})(R .(y) \otimes \mathrm{id}) \sigma \mathrm{r} \\
& =(\mathrm{id} \otimes R .(x))(L .(y) \otimes \mathrm{id}) \sigma \mathrm{r}+(\mathrm{id} \otimes R .(x) R .(y)) \mathrm{r}, \\
(\mathrm{id} \otimes L .(x)) \Delta(y) & =(\mathrm{id} \otimes L .(x))(\mathrm{id} \otimes L .(y)) \mathrm{r}+(\mathrm{id} \otimes L .(x))(R .(y) \otimes \mathrm{id}) \sigma \mathrm{r} \\
& =(\mathrm{id} \otimes L .(x) L .(y)) \mathrm{r}+(\mathrm{id} \otimes L .(x))(R .(y) \otimes \mathrm{id}) \sigma \mathrm{r} .
\end{aligned}
$$

Hence we have

$$
\begin{array}{r}
(R .(y) \otimes \mathrm{id}+\sigma(\mathrm{id} \otimes L .(y))) \Delta(x)+(\mathrm{id} \otimes L .(x)+\sigma(R .(x) \otimes \mathrm{id})) \Delta(y) \\
-\Delta(x \cdot y)-\sigma \Delta(y \cdot x)=(R .(y) \otimes L .(x)+L .(y) \otimes R .(x))(\mathrm{r}+\sigma \mathrm{r}) .
\end{array}
$$

Therefore Eq. (3.7) hold if and only if Eq. (4.3) holds.

(b) Let $x, y \in A$. Then we have

$$
\begin{aligned}
\sigma(\mathrm{id} \otimes R .(y)) \Delta(x) & =\sigma(\mathrm{id} \otimes R .(y))(\mathrm{id} \otimes L .(x)) \mathrm{r}+\sigma(\mathrm{id} \otimes R .(y))(R .(x) \otimes \mathrm{id}) \sigma \mathrm{r} \\
& =(R .(y) L .(x) \otimes \mathrm{id}) \sigma \mathrm{r}+(R .(y) \otimes \mathrm{id})(\mathrm{id} \otimes R .(x)) \mathrm{r}, \\
(\mathrm{id} \otimes R .(y)) \Delta(x) & =(\mathrm{id} \otimes R .(y))(\mathrm{id} \otimes L .(x)) \mathrm{r}+(\mathrm{id} \otimes R .(y))(R .(x) \otimes \mathrm{id}) \sigma \mathrm{r} \\
& =(\mathrm{id} \otimes R .(y) L .(x)) \mathrm{r}+(\mathrm{id} \otimes R .(y))(R .(x) \otimes \mathrm{id}) \sigma \mathrm{r}, \\
\sigma(L .(y) \otimes \mathrm{id}) \Delta(x) & =\sigma(L .(y) \otimes \mathrm{id})(\mathrm{id} \otimes L .(x)) \mathrm{r}+\sigma(L .(y) \otimes \mathrm{id})(R .(x) \otimes \mathrm{id}) \sigma \mathrm{r} \\
& =(\mathrm{id} \otimes L .(y))(L .(x) \otimes \mathrm{id}) \sigma \mathrm{r}+(\mathrm{id} \otimes L .(y) R .(x)) \mathrm{r}, \\
(L .(y) \otimes \mathrm{id}) \Delta(x) & =(L .(y) \otimes \mathrm{id})(\mathrm{id} \otimes L .(x)) \mathrm{r}+(L .(y) \otimes \mathrm{id})(R .(x) \otimes \mathrm{id}) \sigma \mathrm{r} \\
& =(L .(y) \otimes \mathrm{id})(\mathrm{id} \otimes L .(x)) \mathrm{r}+(L .(y) R .(x) \otimes \mathrm{id}) \sigma \mathrm{r} .
\end{aligned}
$$

Therefore we have

$$
\begin{aligned}
& (\sigma(\mathrm{id} \otimes R .(y))+(L .(y) \otimes \mathrm{id})-(\mathrm{id} \otimes R .(y))-\sigma(L .(y) \otimes \mathrm{id})) \Delta(x) \\
& -(\sigma(\mathrm{id} \otimes R .(x))+(L .(x) \otimes \mathrm{id})-(\mathrm{id} \otimes R .(x))-\sigma(L .(x) \otimes \mathrm{id})) \Delta(y) \\
& =(R .(y) \otimes R .(x)+L .(y) \otimes L .(x)-R .(x) \otimes R .(y)-L .(x) \otimes L .(y))(\mathrm{r}+\sigma \mathrm{r}) \\
& +(([L .(y), R .(x)]-[L .(x), R .(y)]) \otimes \mathrm{id}) \sigma \mathrm{r}+(\mathrm{id} \otimes([L .(x), R .(y)]-[L .(y), R .(x)])) \mathrm{r} \\
& =(R .(y) \otimes R .(x)+L .(y) \otimes L .(x)-R .(x) \otimes R .(y)-L .(x) \otimes L .(y))(\mathrm{r}+\sigma \mathrm{r}) .
\end{aligned}
$$

Note that the last equal sign is due to the definition of an anti-flexible algebra. Hence Eq. (3.8) hold if and only if Eq. (4.4) holds.

Lemma 4.3. Let $A$ be a vector space and $\Delta: A \rightarrow A \otimes A$ be a linear map. Then the dual map $\Delta^{*}: A^{*} \otimes A^{*} \rightarrow A^{*}$ defines an anti-flexible algebra structure on $A^{*}$ if and only if $E_{\Delta}=0$, where

$$
E_{\Delta}=(\Delta \otimes \mathrm{id}) \Delta-(\mathrm{id} \otimes \Delta) \Delta+((\sigma \Delta) \otimes \mathrm{id})(\sigma \Delta)-(\mathrm{id} \otimes(\sigma \Delta))(\sigma \Delta) .
$$

Proof. Denote by "०" the product on $A^{*}$ defined by $\Delta^{*}$, that is,

$$
\langle a \circ b, x\rangle=\left\langle\Delta^{*}(a \otimes b), x\right\rangle=\langle a \otimes b, \Delta(x)\rangle \forall x \in A, a, b \in A^{*} .
$$

Therefore, for all $a, b, c \in A^{*}$ and $x \in A$, we have

$$
\begin{aligned}
\langle(a, b, c), x\rangle & =\langle(a \circ b) \circ c-a \circ(b \circ c), x\rangle=\left\langle\left(\Delta^{*}\left(\Delta^{*} \otimes \mathrm{id}\right)-\Delta^{*}\left(\mathrm{id} \otimes \Delta^{*}\right)\right)(a \otimes b \otimes c), x\right\rangle \\
& =\langle((\Delta \otimes \mathrm{id}) \Delta-(\mathrm{id} \otimes \Delta) \Delta)(x), a \otimes b \otimes c\rangle ;
\end{aligned}
$$




$$
\begin{aligned}
\langle(c, b, a), x\rangle & =\langle(c \circ b) \circ a-c \circ(b \circ a), x\rangle=\left\langle\left(\Delta^{*}\left(\Delta^{*} \otimes \mathrm{id}\right)-\Delta^{*}\left(\mathrm{id} \otimes \Delta^{*}\right)\right)(c \otimes b \otimes a), x\right\rangle \\
& =\left\langle\left(\left(\Delta^{*} \sigma^{*}\right)\left(\left(\Delta^{*} \sigma^{*}\right) \otimes \mathrm{id}\right)-\left(\Delta^{*} \sigma^{*}\right)\left(\mathrm{id} \otimes\left(\Delta^{*} \sigma^{*}\right)\right)\right)(a \otimes b \otimes c), x\right\rangle \\
& =\langle(((\sigma \Delta) \otimes \mathrm{id})(\sigma \Delta)-(\mathrm{id} \otimes(\sigma \Delta))(\sigma \Delta))(x), a \otimes b \otimes c\rangle .
\end{aligned}
$$

Therefore, $\left(A^{*}, \circ\right)$ is an anti-flexible algebra if and only if $E_{\Delta}=0$.

Let $(A, \cdot)$ be an anti-flexible algebra and $\mathrm{r}=\sum_{i} a_{i} \otimes b_{i} \in A \otimes A$. Set

$$
\begin{aligned}
& \mathrm{r}_{12}=\sum_{i} a_{i} \otimes b_{i} \otimes 1, \quad \mathrm{r}_{13}=\sum_{i} a_{i} \otimes 1 \otimes b_{i}, \quad r_{23}=\sum_{i} 1 \otimes a_{i} \otimes b_{i}, \\
& \mathrm{r}_{21}=\sum_{i} b_{i} \otimes a_{i} \otimes 1, \quad \mathrm{r}_{31}=\sum_{i} b_{i} \otimes 1 \otimes a_{i}, \quad r_{32}=\sum_{i} 1 \otimes b_{i} \otimes a_{i},
\end{aligned}
$$

where 1 is the unit if $(A, \cdot)$ has a unit, otherwise is a symbol playing a similar role of the unit. Then the operation between two rs is in an obvious way. For example,

$$
\mathrm{r}_{12} \mathrm{r}_{13}=\sum_{i, j} a_{i} \cdot a_{j} \otimes b_{i} \otimes b_{j}, \mathrm{r}_{13} \mathrm{r}_{23}=\sum_{i, j} a_{i} \otimes a_{j} \otimes b_{i} \cdot b_{j}, \mathrm{r}_{23} \mathrm{r}_{12}=\sum_{i, j} a_{j} \otimes a_{i} \cdot b_{j} \otimes b_{i},
$$

and so on.

Theorem 4.4. Let $(A, \cdot)$ be an anti-flexible algebra and $\mathrm{r} \in A \otimes A$. Let $\Delta: A \rightarrow A \otimes A$ be a linear map defined by Eq. (4.1). Then $\Delta^{*}$ defines an anti-flexible algebra structure on $A^{*}$ if and only if for any $x \in A$,

$$
\begin{aligned}
& (\mathrm{id} \otimes \mathrm{id} \otimes L .(x))(M(\mathrm{r}))+(\mathrm{id} \otimes \mathrm{id} \otimes R .(x))(P(\mathrm{r})) \\
& +(L .(x) \otimes \mathrm{id} \otimes \mathrm{id})(N(\mathrm{r}))+(R .(x) \otimes \mathrm{id} \otimes \mathrm{id})(Q(\mathrm{r}))=0,
\end{aligned}
$$

where

$$
\begin{aligned}
& M(\mathrm{r})=\mathrm{r}_{23} \mathrm{r}_{12}+\mathrm{r}_{21} r_{13}-\mathrm{r}_{13} \mathrm{r}_{23}, \quad N(\mathrm{r})=\mathrm{r}_{31} \mathrm{r}_{21}-\mathrm{r}_{21} \mathrm{r}_{32}-\mathrm{r}_{23} \mathrm{r}_{31}, \\
& P(\mathrm{r})=\mathrm{r}_{13} \mathrm{r}_{21}+\mathrm{r}_{12} r_{23}-\mathrm{r}_{23} \mathrm{r}_{13}, \quad Q(\mathrm{r})=\mathrm{r}_{21} \mathrm{r}_{31}-\mathrm{r}_{31} \mathrm{r}_{23}-\mathrm{r}_{32} \mathrm{r}_{21} .
\end{aligned}
$$

Proof. Set $\mathrm{r}=\sum_{i} a_{i} \otimes b_{i}$. Let $x \in A$. Then we have

$$
\begin{aligned}
(\Delta \otimes \mathrm{id}) \Delta(x) & =\sum_{i, j}\left\{a_{j} \otimes\left(a_{i} \cdot b_{j}\right) \otimes\left(x \cdot b_{i}\right)+\left(b_{j} \cdot a_{i}\right) \otimes a_{j} \otimes\left(x \cdot b_{i}\right)\right. \\
& \left.+a_{j} \otimes\left(\left(b_{i} \cdot x\right) b_{j}\right) \otimes a_{i}+\left(b_{j} \cdot\left(b_{i} \cdot x\right)\right) \otimes a_{j} \otimes a_{i}\right\} \\
& =\sum_{i, j}\left\{a_{j} \otimes\left(\left(b_{i} \cdot x\right) \cdot b_{j}\right) \otimes a_{i}+\left(b_{j} \cdot\left(b_{i} \cdot x\right)\right) \otimes a_{j} \otimes a_{i}\right\} \\
& +(\mathrm{id} \otimes \mathrm{id} \otimes L \cdot(x))\left(\mathrm{r}_{23} \mathrm{r}_{12}+\mathrm{r}_{21} \mathrm{r}_{13}\right), \\
((\sigma \Delta) \otimes \mathrm{id})(\sigma \Delta)(x) & =\sum_{i, j}\left\{\left(\left(x \cdot b_{i}\right) \cdot b_{j}\right) \otimes a_{j} \otimes a_{i}+a_{j} \otimes\left(b_{j} \cdot\left(x \cdot b_{i}\right)\right) \otimes a_{i}\right. \\
& \left.+\left(a_{i} \cdot b_{j}\right) \otimes a_{j} \otimes\left(b_{i} \cdot x\right)+a_{j} \otimes\left(b_{j} \cdot a_{i}\right) \otimes\left(b_{i} \cdot x\right)\right\} \\
& =\sum_{i, j}\left\{\left(\left(x \cdot b_{i}\right) \cdot b_{j}\right) \otimes a_{j} \otimes a_{i}+a_{j} \otimes\left(b_{j} \cdot\left(x \cdot b_{i}\right)\right) \otimes a_{i}\right\} \\
& +(\mathrm{id} \otimes \mathrm{id} \otimes R .(x))\left(\mathrm{r}_{13} \mathrm{r}_{21}+\mathrm{r}_{12} \mathrm{r}_{23}\right), \\
& =\sum_{i, j}\left\{a_{i} \otimes a_{j} \otimes\left(\left(x \cdot b_{i}\right) \cdot b_{j}\right)+a_{i} \otimes\left(b_{j} \cdot\left(x \cdot b_{i}\right)\right) \otimes a_{j}\right. \\
& \left.+\left(b_{i} \cdot x\right) \otimes a_{j} \otimes\left(a_{i} \cdot b_{j}\right)+\left(b_{i} \cdot x\right) \otimes\left(b_{j} \cdot a_{i}\right) \otimes a_{j}\right\} \\
& =\sum_{i, j}\left\{a_{i} \otimes a_{j} \otimes\left(\left(x \cdot b_{i}\right) \cdot b_{j}\right)+a_{i} \otimes\left(b_{j} \cdot\left(x \cdot b_{i}\right)\right) \otimes a_{j}\right\}
\end{aligned}
$$




$$
\begin{aligned}
(\mathrm{id} \otimes(\sigma \Delta))(\sigma \Delta)(x) & =\sum_{i, j}\left\{\left(x \cdot b_{i}\right) \otimes\left(a_{i} \cdot b_{j}\right) \otimes a_{j}+\left(x \cdot b_{i}\right) \otimes a_{j} \otimes\left(b_{j} \cdot a_{i}\right)\right. \\
& \left.+a_{i} \otimes\left(\left(b_{i} \cdot x\right) \cdot b_{j}\right) \otimes a_{j}+a_{i} \otimes a_{j} \otimes\left(b_{j} \cdot\left(b_{i} \cdot x\right)\right)\right\} \\
& =\sum_{i, j}\left\{a_{i} \otimes\left(\left(b_{i} \cdot x\right) \cdot b_{j}\right) \otimes a_{j}+a_{i} \otimes a_{j} \otimes\left(b_{j} \cdot\left(b_{i} \cdot x\right)\right)\right\} \\
& +(L .(x) \otimes \mathrm{id} \otimes \mathrm{id})\left(\mathrm{r}_{21} \mathrm{r}_{32}+\mathrm{r}_{23} \mathrm{r}_{31}\right) .
\end{aligned}
$$

Thus $E_{\Delta}(x)=(A 1)+(A 2)+(A 3)$, where

$$
\begin{aligned}
(A 1) & =(\mathrm{id} \otimes \mathrm{id} \otimes L .(x))\left(\mathrm{r}_{23} \mathrm{r}_{12}+\mathrm{r}_{21} \mathrm{r}_{13}\right)+(\mathrm{id} \otimes \mathrm{id} \otimes R .(x))\left(\mathrm{r}_{13} \mathrm{r}_{21}+\mathrm{r}_{12} \mathrm{r}_{23}\right) \\
& -(R .(x) \otimes \mathrm{id} \otimes \mathrm{id})\left(\mathrm{r}_{31} \mathrm{r}_{23}+\mathrm{r}_{32} \mathrm{r}_{21}\right)-(L .(x) \otimes \mathrm{id} \otimes \mathrm{id})\left(\mathrm{r}_{21} \mathrm{r}_{32}+\mathrm{r}_{23} \mathrm{r}_{31}\right), \\
(A 2) & =\sum_{i, j}\left\{a_{j} \otimes\left(\left(b_{i} \cdot x\right) \cdot b_{j}+b_{j} \cdot\left(x \cdot b_{i}\right)\right) \otimes a_{i}-a_{i} \otimes\left(b_{j} \cdot\left(x \cdot b_{i}\right)+\left(b_{i} \cdot x\right) \cdot b_{j}\right) \otimes a_{j}\right\}, \\
(A 3) & =\sum_{i, j}\left\{\left(\left(x \cdot b_{i}\right) \cdot b_{j}+b_{j} \cdot\left(b_{i} \cdot x\right)\right) \otimes a_{j} \otimes a_{i}-a_{i} \otimes a_{j} \otimes\left(\left(x \cdot b_{i}\right) \cdot b_{j}+b_{j} \cdot\left(b_{i} \cdot x\right)\right)\right\} .
\end{aligned}
$$

By exchanging the indices $i$ and $j$, we have $(A 2)=0$.

By the definition of an anti-flexible algebra, we have

$$
\begin{aligned}
(A 3) & =\sum_{i, j}\left\{\left(x \cdot\left(b_{i} \cdot b_{j}\right)+\left(b_{j} \cdot b_{i}\right) \cdot x\right) \otimes a_{j} \otimes a_{i}-a_{i} \otimes a_{j} \otimes\left(x \cdot\left(b_{i} \cdot b_{j}\right)+\left(b_{j} \cdot b_{i}\right) \cdot x\right)\right\} \\
& =(L .(x) \otimes \mathrm{id} \otimes \mathrm{id})\left(\mathrm{r}_{31} \mathrm{r}_{21}\right)+(R .(x) \otimes \mathrm{id} \otimes \mathrm{id})\left(\mathrm{r}_{21} \mathrm{r}_{31}\right) \\
& -(\mathrm{id} \otimes \mathrm{id} \otimes R .(x))\left(\mathrm{r}_{23} \mathrm{r}_{13}\right)-(\mathrm{id} \otimes \mathrm{id} \otimes L .(x))\left(\mathrm{r}_{13} \mathrm{r}_{23}\right) .
\end{aligned}
$$

Then we have

$$
\begin{aligned}
E_{\Delta}(x) & =(\mathrm{id} \otimes \mathrm{id} \otimes L .(x))\left(\mathrm{r}_{23} \mathrm{r}_{12}+\mathrm{r}_{21} \mathrm{r}_{13}-\mathrm{r}_{13} \mathrm{r}_{23}\right)+(\mathrm{id} \otimes \mathrm{id} \otimes R .(x))\left(\mathrm{r}_{13} \mathrm{r}_{21}+\mathrm{r}_{12} \mathrm{r}_{23}-\mathrm{r}_{23} \mathrm{r}_{13}\right) \\
& -(R .(x) \otimes \mathrm{id} \otimes \mathrm{id})\left(\mathrm{r}_{31} \mathrm{r}_{23}+\mathrm{r}_{32} \mathrm{r}_{21}-\mathrm{r}_{21} \mathrm{r}_{31}\right)-(L .(x) \otimes \mathrm{id} \otimes \mathrm{id})\left(\mathrm{r}_{21} \mathrm{r}_{32}+\mathrm{r}_{23} \mathrm{r}_{31}-\mathrm{r}_{31} \mathrm{r}_{21}\right) .
\end{aligned}
$$

Hence the conclusion follows.

Remark 4.5. In fact, for any $\mathrm{r} \in A \otimes A$, we have

$$
N(\mathrm{r})=-\sigma_{13} M(\mathrm{r}), \quad P(\mathrm{r})=\sigma_{12} M(\mathrm{r}), \quad Q(\mathrm{r})=-\sigma_{12} \sigma_{13} M(\mathrm{r}),
$$

where $\sigma_{12}(x \otimes y \otimes z)=y \otimes x \otimes z, \sigma_{13}(x \otimes y \otimes z)=z \otimes y \otimes x$, for any $x, y, z \in A$. sion.

Combing Proposition 4.2, Theorem 4.4 and Remark 4.5 together, we have the following conclu-

Theorem 4.6. Let $(A, \cdot)$ be an anti-flexible algebra and $\mathrm{r} \in A \otimes A$. Let $\Delta: A \rightarrow A \otimes A$ be a linear map defined by Eq. (4.1). Then $(A, \Delta)$ is an anti-flexible bialgebra if and only if $r$ satisfies Eqs. (4.3), (4.4) and

$$
\begin{aligned}
& \left((\mathrm{id} \otimes \mathrm{id} \otimes L .(x))-(R .(x) \otimes \mathrm{id} \otimes \mathrm{id}) \sigma_{12} \sigma_{13}\right. \\
& +\left((\mathrm{id} \otimes \mathrm{id} \otimes R .(x)) \sigma_{12}-(L .(x) \otimes \mathrm{id} \otimes \mathrm{id}) \sigma_{13}\right) M(\mathrm{r})=0,
\end{aligned}
$$

where $M(\mathrm{r})=\mathrm{r}_{23} \mathrm{r}_{12}+\mathrm{r}_{21} \mathrm{r}_{13}-\mathrm{r}_{13} \mathrm{r}_{23}$.

As a direct consequence of Theorem 1.6, we have the following result.

Corollary 4.7. Let $(A, \cdot)$ be an anti-flexible algebra and $\mathrm{r} \in A \otimes A$. Let $\Delta: A \rightarrow A \otimes A$ be a linear map defined by Eq. (4.1). If in addition, $\mathrm{r}$ is skew-symmetric and $\mathrm{r}$ satisfies

$$
\mathrm{r}_{12} \mathrm{r}_{13}-\mathrm{r}_{23} \mathrm{r}_{12}+\mathrm{r}_{13} \mathrm{r}_{23}=0
$$

then $(A, \Delta)$ is an anti-flexible bialgebra. 
Remark 4.8. In fact, there is certain "freedom degree" for the construction of $\Delta: A \rightarrow A \otimes A$ defined by Eq. (4.1). Explicitly, assume

$$
\Delta^{\prime}(x)=\Delta(x)+\Pi(x)(\mathrm{r}+\sigma(\mathrm{r}))=(\mathrm{id} \otimes L .(x)+\Pi(x)) \mathrm{r}+(R .(x) \otimes \mathrm{id}+\Pi(x)) \sigma(\mathrm{r}), \quad \forall x \in A,
$$

where $\Pi(x)$ is an operator depending on $x$ acting on $A \otimes A$. Then by a direct and similar proof as of Theorem 4.6 or by Theorem 4.6 through the relationship between $\Delta^{\prime}$ and $\Delta$, one can show that $\left(A, \Delta^{\prime}\right)$ is an anti-flexible bialgebra if and only if the following equations hold:

LHS of Eq. $(4.3)+A(x)(\mathrm{r}+\sigma(\mathrm{r}))=0$,

LHS of Eq. $(4.4)+B(x)(\mathrm{r}+\sigma(\mathrm{r}))=0$,

LHS of Eq. (4.10) $+C_{12}(x)\left(\mathrm{r}_{12}+\mathrm{r}_{21}\right)+C_{23}(x)\left(\mathrm{r}_{23}+\mathrm{r}_{32}\right)+C_{13}(x)\left(\mathrm{r}_{13}+\mathrm{r}_{31}\right)=0$,

where $A(x), B(x)$ are operators depending on $x$ acting on $A \otimes A, C_{12}(x), C_{23}(x), C_{13}(x)$ are operators depending on $x$ acting on $A \otimes A \otimes A$ (it is the component itself when the component acts on 1 ), and all of them are related to $\Pi(x)$. Hence by this conclusion (which is independent of Theorem 1.6) directly, we still show that $\left(A, \Delta^{\prime}\right)$ is an anti-flexible bialgebra when $\mathrm{r}$ is skew-symmetric and $\mathrm{r}$ satisfies Eq. (4.11). That is, this $\Delta^{\prime}$ defined by Eq. (4.12), also leads to the introduction of Eq. (4.11). Note that when $\mathrm{r}$ is skew-symmetric, $\Delta^{\prime}=\Delta$.

Definition 4.9. Let $(A, \cdot)$ be an anti-flexible algebra and $\mathrm{r} \in A \otimes A$. Eq. (4.11) is called the anti-flexible Yang-Baxter equation $(\mathrm{AFYBE})$ in $(A, \cdot)$.

Remark 4.10. The notion of anti-flexible Yang-Baxter equation in an anti-flexible algebra is due to the fact that it is an analogue of the classical Yang-Baxter equation in a Lie algebra ([9]) or the associative Yang-Baxter equation in an associative algebra ([3]).

It is a remarkable observation and an unexpected consequence that both the anti-flexible YangBaxter equation in an anti-flexible algebra and the associative Yang-Baxter equation ([3]) in an associative algebra have the same form Eq. (4.11). Hence both these two equations have some common properties. At the end of this section, we give two properties of anti-flexible Yang-Baxter equation whose proofs are omitted since the proofs are the same as in the case of associative Yang-Baxter equation.

Let $A$ be a vector space. For any $\mathrm{r} \in A \otimes A, \mathrm{r}$ can be regarded as a linear map from $A^{*}$ to $A$ in the following way:

$$
\left\langle\mathrm{r}, u^{*} \otimes v^{*}\right\rangle=\left\langle\mathrm{r}\left(u^{*}\right), v^{*}\right\rangle, \quad \forall u^{*}, v^{*} \in A^{*} .
$$

Proposition 4.11. Let $(A, \cdot)$ be an anti-flexible algebra and $\mathrm{r} \in A \otimes A$ be skew-symmetric. Then $\mathrm{r}$ is solution of anti-flexible Yang-Baxter equation if and only if $\mathrm{r}$ satisfies

$$
\mathrm{r}(a) \cdot \mathrm{r}(b)=\mathrm{r}\left(R_{*}^{*}(\mathrm{r}(a)) b+L_{.}^{*}(\mathrm{r}(b)) a\right), \quad \forall a, b \in A^{*} .
$$

Remark 4.12. Since the dual bimodules of both anti-flexible and associative algebras have the same form (see Remark 2.8), the interpretation of anti-flexible Yang-Baxter equation in terms of operator form (4.14) in the above Proposition 4.11 explains partly why the anti-flexible YangBaxter equation has the same form as of the associative Yang-Baxter equation.

Theorem 4.13. Let $(A, \cdot)$ be an anti-flexible algebra and $\mathrm{r} \in A \otimes A$. Suppose that $\mathrm{r}$ is antisymmetric and nondegenerate. Then $\mathrm{r}$ is a solution of anti-flexible Yang-Baxter equation in $(A, \cdot)$ if and only if the inverse of the isomorphism $A^{*} \rightarrow A$ induced by $\mathrm{r}$, regarded as a bilinear form $\omega$ on $A$ (that is, $\omega(x, y)=\left\langle\mathrm{r}^{-1} x, y\right\rangle$ for any $\left.x, y \in A\right)$, satisfies

$$
\omega(x \cdot y, z)+\omega(y \cdot z, x)+\omega(z \cdot x, y)=0, \quad \forall x, y, z \in A .
$$




\section{O-OPERATORS OF ANTI-FLEXIBLE ALGEBRAS AND PRE-ANTI-FLEXIBLE ALGEBRAS}

In this section, we introduce the notions of $\mathcal{O}$-operators of anti-flexible algebras and pre-antiflexible algebras to construct skew-symmetric solutions of anti-flexible Yang-Baxter equation and hence to construct anti-flexible bialgebras.

Definition 5.1. Let $(l, r, V)$ be a bimodule of an anti-flexible algebra $(A, \cdot)$. A linear map $T$ : $V \rightarrow A$ is called an $\mathcal{O}$-operator associated to $(l, r, V)$ if $T$ satisfies

$$
T(u) \cdot T(v)=T(l(T(u)) v+r(T(v)) u), \quad \forall u, v \in V .
$$

Example 5.2. Let $(A, \cdot)$ be an anti-flexible algebra. An $\mathcal{O}$-operator $R_{B}$ associated to the regular bimodule $(L, R, A)$ is called a Rota-Baxter operator of weight zero, that is, $R_{B}$ satisfies

$$
R_{B}(x) \cdot R_{B}(y)=R_{B}\left(R_{B}(x) \cdot y+x \cdot R_{B}(y)\right), \quad \forall x, y \in A .
$$

Example 5.3. Let $(A, \cdot)$ be an anti-flexible algebra and $\mathrm{r} \in A \otimes A$. If $\mathrm{r}$ is skew-symmetric, then by Proposition 1.11, $\mathrm{r}$ is a solution of anti-flexible Yang-Baxter equation if and only if $\mathrm{r}$ regarded as a linear map from $A^{*}$ to $A$ is an $\mathcal{O}$-operator associated to the bimodule $\left(R_{.}^{*}, L_{.}^{*}, A^{*}\right)$.

There is the following construction of (skew-symmetric) solutions of anti-flexible Yang-Baxter equation in a semi-direct product anti-flexible algebra from an $\mathcal{O}$-operator of an anti-flexible algebra which is similar as for associative algebras ([3, Theorem 2.5.5], hence the proof is omitted).

Theorem 5.4. Let $(l, r, V)$ be a bimodule of an anti-flexible algebra $(A, \cdot)$. Let $T: V \rightarrow A$ be a linear map which is identified as an element in $\left(A \ltimes_{r^{*}, l^{*}} V^{*}\right) \oplus\left(A \ltimes_{r^{*}, l^{*}} V^{*}\right)$. Then $\mathrm{r}=T-\sigma(T)$ is a skew-symmetric solution of anti-flexible Yang-Baxter equation in $A \ltimes_{r^{*}, l^{*}} V^{*}$ if only if $T$ is an $\mathcal{O}$-operator associated to the bimodule $(l, r, V)$.

Definition 5.5. Let $A$ be a vector space with two bilinear products $\prec, \succ: A \otimes A \rightarrow A$. We call it a pre-anti-flexible algebra denoted by $(A, \prec, \succ)$ if for any $x, y, z \in A$, the following equations are satisfied

$$
\begin{aligned}
(x, y, z)_{m} & =(z, y, x)_{m} \\
(x, y, z)_{l} & =(z, y, x)_{r}
\end{aligned}
$$

where

$$
\begin{aligned}
(x, y, z)_{m} & :=(x \succ y) \prec z-x \succ(y \prec z), \\
(x, y, z)_{l} & :=(x * y) \succ z-x \succ(y \succ z), \\
(x, y, z)_{r} & :=(x \prec y) \prec z-x \prec(y * z),
\end{aligned}
$$

here $x * y=x \prec y+x \succ y$.

Remark 5.6. Note that if both hand sides in Eqs. (5.5), (5.6) and (5.7) are zero, that is,

$$
(x, y, z)_{m}=0, \quad(z, y, x)_{l}=0, \quad(x, y, z)_{r}=0,
$$

then it exactly gives the definition of a dendriform algebra which was introduced by Loday in [15]. Hence any dendriform algebra is a pre-anti-flexible algebra, that is, pre-anti-flexible algebras can be regarded as a natural generalization of dendriform algebras. On the other hand, from the point of view of operads, like dendriform algebras being the splitting of associative algebras, pre-anti-flexible algebras are the splitting of anti-flexible algebras ([4, [] 6 ). 
Proposition 5.7. Let $(A, \prec, \succ)$ be a pre-anti-flexible algebra. Define a bilinear product $*: A \otimes$ $A \rightarrow A$ by

$$
x * y=x \prec y+x \succ y, \quad \forall x, y \in A .
$$

Then $(A, *)$ is an anti-flexible algebra, which is called the associated anti-flexible algebra of $(A, \prec, \succ)$.

Proof. Set

$$
(x, y, z)_{*}=(x * y) * z-x *(y * z), \quad \forall x, y, z \in A .
$$

Then for any $x, y, z \in A$, we have

$$
(x, y, z)_{*}=(x, y, z)_{m}+(x, y, z)_{l}+(x, y, z)_{r}=(z, y, x)_{m}+(z, y, x)_{l}+(z, y, x)_{r}=(z, y, x)_{*} .
$$

Hence $(A, *)$ is an anti-flexible algebra.

Let $(A, \prec, \succ)$ be a pre-anti-flexible algebra. For any $x \in A$, let $L_{\succ}(x), R_{\prec}(x)$ denote the left multiplication operator of $(A, \prec)$ and the right multiplication operator of $(A, \succ)$ respectively, that is, $L_{\succ}(x)(y)=x \succ y, \quad R_{\prec}(x)(y)=y \prec x, \quad \forall x, y \in A$. Moreover, let $L_{\succ}, R_{\prec}: A \rightarrow \mathfrak{g l}(A)$ be two linear maps with $x \rightarrow L_{\succ}(x)$ and $x \rightarrow R_{\prec}(x)$ respectively.

Proposition 5.8. Let $(A, \prec, \succ)$ be a pre-anti-flexible algebra. Then $\left(L_{\succ}, R_{\prec}, A\right)$ is a bimodule of the associated anti-flexible algebra $(A, *)$, where $*$ is defined by Eq. (5.9).

Proof. For any $x, y, z \in A$, we have

$$
\begin{aligned}
& \left(L_{\succ}(x * y)-L_{\succ}(x) L_{\succ}(y)\right)(z)=(x * y) \succ z-x \succ(y \succ z)=(x, y, z)_{l}, \\
& \left(R_{\prec}(x) R_{\prec}(y)-R_{\prec}(y * x)\right)(z)(z \prec y) \prec x-z \prec(y * x)=(z, y, x)_{r}, \\
& \left(L_{\succ}(x) R_{\prec}(y)-R_{\prec}(y) L_{\succ}(x)\right)(z)=x \succ(z \prec y)-(x \succ z) \prec y=(x, z, y)_{m}, \\
& \left(L_{\succ}(y) R_{\prec}(x)-R_{\prec}(x) L_{\succ}(y)\right)(z)=y \succ(z \prec x)-(y \succ z) \prec x=(y, z, x)_{m} .
\end{aligned}
$$

Hence $\left(L_{\succ}, R_{\prec}, A\right)$ is a bimodule of $(A, *)$.

A direct consequence is given as follows.

Corollary 5.9. Let $(A, \prec, \succ)$ be a pre-anti-flexible algebra. Then the identity map id is an $\mathcal{O}$ operator of the associated anti-flexible algebra $(A, *)$ associated to the bimodule $\left(L_{\succ}, R_{\prec}, A\right)$.

Theorem 5.10. Let $(l, r, V)$ be a bimodule of an anti-flexible algebra $(A, \cdot)$. Let $T: V \rightarrow A$ be an $\mathcal{O}$-operator associated to $(l, r, V)$. Then there exists a pre-anti-flexible algebra structure on $V$ given by

$$
u \succ v=l(T(u)) v, \quad u \prec v=r(T(v)) u, \quad \forall u, v \in V .
$$

So there is an associated anti-flexible algebra structure on $V$ given by Eq. (5.9) and $T$ is a homomorphism of anti-flexible algebras. Moreover, $T(V)=\{T(v) \mid v \in V\} \subset A$ is an anti-flexible subalgebra of $(A, \cdot)$ and there is an induced pre-anti-flexible algebra structure on $T(V)$ given by

$$
T(u) \succ T(v)=T(u \succ v), \quad T(u) \prec T(v)=T(u \prec v), \quad \forall u, v \in V .
$$

Its corresponding associated anti-flexible algebra structure on $T(V)$ given by Eq. (5.9) is just the anti-flexible subalgebra structure of $(A, \cdot)$ and $T$ is a homomorphism of pre-anti-flexible algebras.

Proof. For all $u, v, w \in V$, we have

$$
\begin{aligned}
(u, v, w)_{m} & =(u \succ v) \prec w-u \succ(v \prec w)=r(T(w)) l(T(u)) v-l(T(u)) r(T(w)) v \\
& =r(T(u)) l(T(w)) v-l(T(u)) r(T(w)) v=(w, v, u)_{m}, \\
(u, v, w)_{l} & =(u \succ v+u \prec v) \succ w-u \succ(v \succ w)=(l(T(l(T(u)) v+r(T(v)) u))-l(T(u)) l(T(v))) w \\
& =(l(T(u) \cdot T(v))-l(T(u)) l(T(v))) w=(r(T(u)) r(T(v))-r(T(v) \cdot T(u)) w
\end{aligned}
$$




$$
\begin{aligned}
& =(r(T(u)) r(T(v))-r(T(u \succ v+u \prec v))) w=(w \prec v) \prec u-w \prec(u \succ v+u \prec v) \\
& =(w, v, u)_{r}
\end{aligned}
$$

Therefore, $(V, \prec, \succ)$ is a pre-anti-flexible algebra. For $T(V)$, we have

$$
T(u) * T(v)=T(u \succ v+u \prec v)=T(u * v)=T(u) \cdot T(v), \quad \forall u, v \in V .
$$

The rest is straightforward.

Corollary 5.11. Let $(A, \cdot)$ be an anti-flexible algebra. Then there exists a pre-anti-flexible algebras structure on $A$ such that its associated anti-flexible algebra is $(A, \cdot)$ if and only if there exists an invertible $\mathcal{O}$-operator.

Proof. Suppose that there exists an invertible $\mathcal{O}$-operator $T: V \rightarrow A$ associated to a bimodule $(l, r, V)$. Then the products " $\succ, \prec$ " given by Eq. (5.10) defines a pre-anti-flexible algebra structure on $V$. Moreover, there is a pre-anti-flexible algebra structure on $T(V)=A$ given by Eq. (5.11), that is,

$$
x \succ y=T\left(l(x) T^{-1}(y)\right), \quad x \prec y=T\left(r(y) T^{-1}(x)\right), \quad \forall x, y \in A .
$$

Moreover, for any $x, y \in A$, we have

$$
x \succ y+x \prec y=T\left(l(x) T^{-1}(y)+r(y) T^{-1}(x)\right)=T\left(T^{-1}(x)\right) \cdot T\left(T^{-1}(y)\right)=x \cdot y .
$$

Hence the associated anti-flexible algebra of $(A, \succ, \prec)$ is $(A, \cdot)$.

Conversely, let $(A, \succ, \prec)$ be pre-anti-flexible algebra such that its associated anti-flexible is $(A, \cdot)$. Then by Corollary 5.9, the identity map id is an $\mathcal{O}$-operator of $(A, \cdot)$ associated to the bimodule $\left(L_{\succ}, R_{\prec}, A\right)$.

Corollary 5.12. Let $(A, \cdot)$ be an anti-flexible algebra and $\omega$ be a nondegenerate skew-symmetric bilinear form satisfying Eq. (4.15). Then there exists a pre-anti-flexible algebra structure $\succ, \prec$ on A given by

$$
\omega(x \succ y, z)=\omega(y, z \cdot x), \omega(x \prec y, z)=\omega(x, y \cdot z), \quad \forall x, y, z \in A,
$$

such that the associated anti-flexible algebra is $(A, \cdot)$.

Proof. Define a linear map $T: A \rightarrow A^{*}$ by

$$
\langle T(x), y\rangle=\omega(x, y), \quad \forall x, y \in A .
$$

Then $T$ is invertible and $T^{-1}$ is an $\mathcal{O}$-operator of the anti-flexible algebra $(A, \cdot)$ associated to the bimodule $\left(R_{*}^{*}, L_{.}^{*}, A^{*}\right)$. By Corollary 5.11, there is a pre-anti-flexible algebra structure $\succ, \prec$ on $(A, *)$ given by

$$
x \succ y=T^{-1} R^{*}(x) T(y), \quad x \prec y=T^{-1} L^{*}(y) T(x), \quad \forall x, y \in A,
$$

which gives exactly Eq. (5.12) such that the associated anti-flexible algebra is $(A, \cdot)$.

Finally we give the following construction of skew-symmetric solutions of anti-flexible YangBaxter equation (hence anti-flexible bialgebras) from a pre-anti-flexible algebra.

Proposition 5.13. Let $(A, \succ, \prec)$ be a pre-anti-flexible algebra. Then

$$
\mathrm{r}=\sum_{i}^{n}\left(e_{i} \otimes e_{i}^{*}-e_{i}^{*} \otimes e_{i}\right)
$$

is a solution of anti-flexible Yang-Baxter equation in $A \ltimes_{R_{\prec}^{*}, L_{\succ}^{*}} A^{*}$, where $\left\{e_{1}, \cdots, e_{n}\right\}$ is a basis of $A$ and $\left\{e_{1}^{*}, \cdots, e_{n}^{*}\right\}$ is its dual basis. 
Proof. Note that the identity map id $=\sum_{i=1}^{n} e_{i} \otimes e_{i}^{*}$. Hence the conclusion follows from Theorem 5.4 and Corollary 5.9.

Acknowledgements. This work is supported by NSFC (11931009). C. Bai is also supported by the Fundamental Research Funds for the Central Universities and Nankai ZhiDe Foundation.

\section{REFERENCES}

[1] M. Aguiar, On the associative analog of Lie bialgebras, J. Algebra, 244, (2001), pp. 492-532. 2

[2] C. Bai, Left-symmetric bialgebras and an analogue of the classical Yang-Baxter equation, Commun. Contemp. Math., 10, (2008), pp. 221-260. 目

[3] C. Bai, Double constructions of Frobenius algebras, Connes cocycles and their duality, J. Noncommut. Geom., 4, (2010), pp. 475-530. 月, 月, 12, 13

[4] C. Bai, O. Bellier, L. Guo and X. Ni, Spliting of operations, Manin products and Rota-Baxter operators, Int. Math. Res. Not., 2013 (2013), pp. 485-524. 3, 13

[5] G.M. Benkart and L.M. Osborn, Flexible Lie-admissible algebra, J. Algebra, 71, (1981), pp. 11-31. 1

[6] M.C. Bhandari, On the classification of simple anti-flexible algebras, Trans. Amer. Math. Soc., 173, (1972), pp. 159-181. 2

[7] H.A. Çelik, On primitive and prime anti-flexible ring, J. Algebra, 20, (1972), pp. 428-440. 2

[8] L.W. Davis and D.J. Rodabaugh, Simple nearly anti-flexible algebras have unity elements, London Math. Soc. Stud. Texts., 2, (1969), pp. 69-72. 目

[9] V.G. Drinfeld, Hamiltonian structures of Lie groups, Lie bialgebras and the geometric meaning of the classical Yang-Baxter equations, Soviet Math. Dokl., 27, (1983), pp. 68-71. 月, 12

[10] M.E. Goncharov, The classical Yang-Baxter equation on alternative algebras: The alternative D-bialgebra structure on the Cayley-Dickson matrix algebra, Sibirsk. Math. Zh., 48, (2007), pp. 1008-1024 (in Russian); translated in Siberian Math. J., 48, (2007), pp. 809-823. 目

[11] M. Goze and E. Remm, Lie-admissible algebras and operads, J. Algebra, 273, (2004), pp. 129-152. 2, 6

[12] M.N. Hounkonnou and M.L. Dassoundo, Center-symmetric algebras and bialgebras: relevant properties and consequences, Trends Math., (2016), pp. 261-273. 2, 目

[13] B.A. Kupershmidt, What a classical $r$-matrix really is, J. Nonlinear. Math. Phys., 6, (1999), pp. 448-488.

[14] F. Kosier, On a class of nonflexible algebras, Trans. Amer. Math. Soc., 102, (1962), pp. 299-318. 1

[15] J.-L. Loday, Dialgebras and related operads, Lecture Notes Math., 1763, Springer, Berlin 2001, pp. 7-66. 月, 13

[16] J. Pei, C. Bai and L. Guo, Splitting of operads and Rota-Baxter operators on operads, Appl. Cate. Stru., 25 (2017), pp. 505-538. B, 13

[17] D.J. Rodabaugh, A generalization of the flexible law, Trans. Amer. Math. Soc., 114, (1965), 468-487.

[18] D.J. Rodabaugh, On semisimple antiflexible algebras, Portugal. Math., 6, (1967), pp. 261-271. 2

[19] D.J. Rodabaugh, On anti-flexible algebras, Trans. Amer. Math. Soc., 169, (1972), pp. 219-235.

[20] D.J. Rodabaugh, A theorem of semisimple anti-flexible algebras, Comm. Algebra, 6, (1978), pp. 1081-1090. 2

[21] V.N. Zhelyabin, Jordan bialgebras and their relation to Lie bialgebras, Algebra Logic, 36, (1997), pp. 1-15. 2

${ }^{\star}$ ) Chern Institute of Mathematics \& LPMC, Nankai University, Tianjin 300071, China

E-mail address: dassoundo@yahoo.com

$\left(^{\dagger}\right)$ Chern Institute of Mathematics \& LPMC, Nankai University, Tianjin 300071, China

E-mail address: baicm.nankai.edu.cn

$\left(^{\ddagger}\right)$ University of Abomey-Calavi, International Chair in Mathematical Physics and Applications, ICMPA-UNESCO Chair, 072 BP 50, Cotonou, ReP. OF BEnin

E-mail address: hounkonnou@yahoo.fr 